\title{
Transversality of isotropic projections, unrectifiability, and Heisenberg groups
}

\author{
Risto Hovila
}

\begin{abstract}
We show that the family of $m$-dimensional isotropic projections in $\mathbb{R}^{2 n}$ is transversal. As an application we show that the BesicovitchFederer projection theorem holds for isotropic projections. We also use transversality to obtain almost sure estimates on the Hausdorff dimension of isotropic projections of subsets $E \subset \mathbb{R}^{2 n}$. These results may also be applied to gain information on the horizontal projections of the Heisenberg group $\mathbb{H}^{n}$.
\end{abstract}

\section{Introduction}

The behaviour of different dimensions under projection-type mappings has been studied for decades. The study was initiated by Marstrand in 1954, when he proved a well-known theorem according to which the Hausdorff dimension of a planar set is preserved under typical orthogonal projections. Over the decades this result has been extended in different directions by many authors. For a detailed survey of a variety of related developments see [7]. In 1968 Kaufman reproved Marstrand's theorem using potential theoretic methods. He also provided an estimate on the size of the set of exceptional directions. Mattila generalized Marstrand's result to higher dimensions in 1975. The wide investigation of related topics culminated in the work of Peres and Schlag, [9]. They introduced a very general formalism for transversal mappings and proved dimension conservation results analogous to Marstrand's theorem. Mattila's generalization of Marstrand's theorem together with Kaufman's exceptional set estimate is actually a special case of the results in [9]. In this paper we show that the family of isotropic projections in $\mathbb{R}^{2 n}$ satisfies a transversality condition. From this result we draw several corollaries both in $\mathbb{R}^{2 n}$ and in the Heisenberg group $\mathbb{H}^{n}$.

Let $\mathbb{R}^{2 n}$ be equipped with the standard symplectic form $\omega: \mathbb{R}^{2 n} \times \mathbb{R}^{2 n} \rightarrow$ $\mathbb{R}, \omega(x, y)=\sum_{i=1}^{n} x_{i+n} y_{i}-x_{i} y_{i+n}$. In this note we examine properties of $m$-di-

Mathematics Subject Classification (2010): Primary 28A80; Secondary 28A78, 53D05.

Keywords: Projection, symplectic geometry, Hausdorff dimension, Heisenberg group, unrectifiability. 
mensional isotropic projections in $\mathbb{R}^{2 n}$, that is, orthogonal projections onto $m$-dimensional isotropic subspaces of $\mathbb{R}^{2 n}$. A linear subspace $V \subset \mathbb{R}^{2 n}$ is isotropic if $\omega(v, w)=0$ for all $v, w \in V$. Isotropic subspaces are closely related to horizontal subgroups of the Heisenberg group $\mathbb{H}^{n}$. Indeed, $\mathbb{V} \subset \mathbb{H}^{n}$ is a horizontal subgroup if and only if $\mathbb{V}=V \times\{0\}$ for some isotropic subspace $V \subset \mathbb{H}^{n}$. Thus the study of isotropic projections also yields results about the horizontal projections of $\mathbb{H}^{n}$.

Our main result is the following theorem.

Theorem 1.1. Let $n$ and $m$ be integers such that $0<m \leq n$, let $G_{h}(n, m)$ be the submanifold of the Grassmannian $G(2 n, m)$ consisting of all isotropic subspaces of $\mathbb{R}^{2 n}$, and denote by $P_{V}: \mathbb{R}^{2 n} \rightarrow V$ the orthogonal projection onto the $m$-plane $V \in G_{h}(n, m)$. Then the projection family $\left\{P_{V}: \mathbb{R}^{2 n} \rightarrow V\right\}_{V \in G_{h}(n, m)}$ is transversal.

There are several notions of transversality in mathematics. Here transversality is understood as in the paper [9] of Peres and Schlag, and it will be defined in Section 3.

This paper is organized as follows. In Section 2 we introduce the basic definitions and notation. In Section 3 we will prove Theorem 1.1. As an application of the main result we give necessary and sufficient conditions under which a subset $E \subset \mathbb{R}^{2 n}$ projects onto a set of measure zero under almost all $m$-dimensional isotropic projections (Theorem 4.1). We also give almost sure dimension estimates on the Hausdorff dimension of isotropic projections of subsets of $\mathbb{R}^{2 n}$ (Theorem 4.3). These estimates were already proven in Theorem 1.2 of [2] using different methods. We improve the result by providing estimates on the dimension of exceptional parameters. The results mentioned above also yield corollaries concerning the dimension of horizontal projections of subsets of the Heisenberg group. These applications will be discussed in Section 4.

\section{Preliminaries}

\subsection{Symplectic geometry}

Let $M$ be a manifold of dimension $2 n$. A symplectic form on $M$ is a closed nondegenerate 2 -form on $M$. The standard form $\omega$ on $\mathbb{R}^{2 n}$ is defined by

$$
\omega(x, y)=\sum_{i=1}^{n} x_{i+n} y_{i}-x_{i} y_{i+n}=(J x \mid y),
$$

where $(\cdot \mid \cdot)$ is the Euclidean inner product on $\mathbb{R}^{2 n}$ and $J$ is the $2 n \times 2 n$-matrix

$$
J=\left(\begin{array}{cc}
0 & I_{n \times n} \\
-I_{n \times n} & 0
\end{array}\right) .
$$

By a well-known theorem of Darboux, every symplectic form on $M$ is locally diffeomorphic to the standard form $\omega$ on $\mathbb{R}^{2 n}$. Furthermore, every symplectic vector space is isomorphic to $\left(\mathbb{R}^{2 n}, \omega\right)$. Below we work only on $\mathbb{R}^{2 n}$ equipped with the standard form $\omega$. For more information on symplectic geometry, see [4]. 
For a linear subspace $V \subset \mathbb{R}^{2 n}$, we define its symplectic orthogonal $V^{\omega}$ by

$$
V^{\omega}=\{w: \omega(w, v)=0 \text { for all } v \in V\} .
$$

A linear subspace $V$ is said to be isotropic if $V \subset V^{\omega}$ and Lagrangian if $V=V^{\omega}$. A subspace $V$ can be Lagrangian only when $\operatorname{dim} V=n$. For integers $0<m \leq n$, we denote by $G(2 n, m)$ the space of all $m$-dimensional linear subspaces of $\mathbb{R}^{2 n}$. It is a compact manifold of dimension $m(2 n-m)$. Furthermore, we define the isotropic Grassmannian $G_{h}(n, m)$ by

$$
G_{h}(n, m)=\left\{V \in G(2 n, m): V \text { is an isotropic subspace of } \mathbb{R}^{2 n}\right\} .
$$

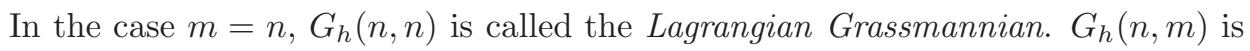
a smooth manifold of dimension $2 n m-m(3 m-1) / 2$, so, for $m>1$, the isotropic Grassmannian $G_{h}(n, m)$ is a submanifold of $G(2 n, m)$ with positive codimension. For $m=1$, these manifolds are the same, $G_{h}(n, m)=G(2 n, m)$. The isotropic Grassmannian can be endowed with a natural measure $\mu_{n, m}$ in a similar way as the usual Grassmannian is endowed with the measure $\gamma_{n, m}$, using unitary instead of orthogonal matrices. See Section 2 of [2] for details.

Next we define local coordinates on the isotropic Grassmannian $G_{h}(n, m)$. We begin by recalling the definition of local coordinates on the Grassmann manifold $G(2 n, m)$. Fix an $m$-plane $V \in G(2 n, m)$ and choose an orthonormal basis $\left\{e_{1}, \ldots, e_{2 n}\right\}$ of $\mathbb{R}^{2 n}$ such that $V=<e_{1}, \ldots, e_{m}>$. Consider all linear maps $L\left(V, V^{\perp}\right)=\left\{L: V \rightarrow V^{\perp}: L\right.$ linear $\}$. The graph $\mathcal{G}(L)=\{(x, L x): x \in V\}$ of any such map is an $m$-plane whose intersection with the $(2 n-m)$-plane $V^{\perp}$ is the zero subspace. Conversely, any $m$-plane with this property is the graph of a unique linear map $L: V \rightarrow V^{\perp}$. Using the basis $\left\{e_{1}, \ldots, e_{m}\right\}$ of $V$ and the basis $\left\{e_{m+1}, \ldots, e_{2 n}\right\}$ for $V^{\perp}, L\left(V, V^{\perp}\right)$ can be identified with $\mathcal{M}(2 n-m, m)$, the space of all $(2 n-m) \times m$ matrices. The $m$-plane associated to a matrix $A=\left(a_{i j}\right) \in \mathcal{M}(2 n-m, m)$ is spanned by the vectors

$$
e_{i}^{A}=e_{i}+\sum_{k=1}^{2 n-m} a_{k i} e_{k+m}, \quad i=1, \ldots, m .
$$

Define a subset $\mathcal{M}_{h}(n, m)$ of all $(2 n-m) \times m$ matrices by

$$
\begin{aligned}
\mathcal{M}_{h}(n, m)=\{ & \left(a_{i j}\right) \in \mathcal{M}(2 n-m, m): a_{(n-m+i) j}=a_{(n-m+j) i} \\
& \left.+\sum_{k=1}^{n-m}\left(a_{k j} a_{(n+k) i}-a_{(n+k) j} a_{k i}\right) \text { for } j<i \leq m, 1 \leq j \leq m\right\} .
\end{aligned}
$$

The independent coordinates $a_{i j}$ in a matrix $\left(a_{i j}\right) \in \mathcal{M}_{h}(n, m)$ are the ones with $j \in\{1, \ldots, m\}$ and $i \in\{1, \ldots, n-m+j, n+1, \ldots, 2 n-m\}$.

$\mathcal{M}_{h}(n, m)$ is an embedded submanifold of $\mathcal{M}(2 n-m, m)$ having dimension $2 n m-m(3 m-1) / 2$. We have the following local parametrization.

Lemma 2.1. The isotropic Grassmannian $G_{h}(n, m)$ can be locally parametrized by the matrices $\mathcal{M}_{h}(n, m)$. 
Proof. Let $V \in G_{h}(n, m)$ and let $\left\{e_{1}, \ldots, e_{2 n}\right\}$ be an orthonormal basis of $\mathbb{R}^{2 n}$ such that $V=<e_{1}, \ldots, e_{m}>, \omega\left(e_{i}, e_{i+n}\right)=1$ for all $i \in\{1, \ldots, n\}$, and $\omega\left(e_{i}, e_{j}\right)=0$ for all other pairs $\left(e_{i}, e_{j}\right)$. It is enough to define the local coordinates only around the $m$-plane $V=<e_{1}, \ldots, e_{m}>$, since the group $U(n)$ of unitary $2 n \times 2 n$-matrices acts transitively on $G_{h}(n, m)$, by Lemma 2.2 of [2], and $\omega(g u, g v)=\omega(u, v)$ for all $u, v \in \mathbb{R}^{2 n}, g \in U(n)$. Let $A \in \mathcal{M}(2 n-m, m)$ and denote the $m$-plane associated to $A$ by $V_{A}$. Then $V_{A} \in G_{h}(n, m)$ if and only if

$$
\omega\left(e_{i}+\sum_{k=1}^{2 n-m} a_{k i} e_{k+m}, e_{j}+\sum_{k=1}^{2 n-m} a_{k j} e_{k+m}\right)=0 \quad \text { for all } i, j=1, \ldots, m
$$

and this is the case precisely when $A \in \mathcal{M}_{h}(n, m)$. Thus we can define coordinates on the isotropic Grassmannian using the matrices $A \in \mathcal{M}_{h}(n, m)$.

\subsection{Heisenberg groups}

For an introduction to Heisenberg groups, see [3]. Below we state the basic facts needed in this paper. The Heisenberg group $\mathbb{H}^{n}$ is the unique simply connected, connected nilpotent Lie group of step two and dimension $2 n+1$ with one-dimensional centre. As a manifold $\mathbb{H}^{n}$ may be identified with $\mathbb{R}^{2 n+1}$. We denote points $p \in \mathbb{H}^{n}$ in coordinates as

$$
p=(z, t)=\left(z_{1}, \ldots, z_{2 n}, t\right) \in \mathbb{R}^{2 n} \times \mathbb{R} .
$$

The group operation is given by

$$
p * p^{\prime}=(z, t) *\left(z^{\prime}, t^{\prime}\right)=\left(z+z^{\prime}, t+t^{\prime}+2 \omega\left(z, z^{\prime}\right)\right),
$$

where $\omega$ is the standard symplectic form on $\mathbb{R}^{2 n}$.

The Heisenberg metric $\mathrm{d}_{\mathrm{H}}$ of $\mathbb{H}^{n}$ can be defined by

$$
\mathrm{d}_{\mathrm{H}}\left(p, p^{\prime}\right):=\left\|p^{-1} * p^{\prime}\right\|_{\mathrm{H}}, \quad \text { where }\|p\|_{\mathrm{H}}:=\left(\|z\|^{4}+t^{2}\right)^{1 / 4} \text {. }
$$

Here $\|\cdot\|$ denotes the Euclidean norm on $\mathbb{R}^{2 n}$. This metric is bi-Lipschitz equivalent to the usual Carnot-Carathéodory metric on $\mathbb{H}^{n}$. The metric $\mathrm{d}_{\mathrm{H}}$ induces the Euclidean topology, but the properties of the metric space $\left(\mathbb{H}^{n}, \mathrm{~d}_{\mathrm{H}}\right)$ differ significantly from those of the underlying Euclidean space. For example, the Hausdorff dimension of $\left(\mathbb{H}^{n}, \mathrm{~d}_{\mathrm{H}}\right)$ is $2 n+2$. Thus, when speaking of the metric properties of $\mathbb{H}^{n}$, we need to specify which metric we are using. We will denote the Hausdorff measure and Hausdorff dimension with respect to the Heisenberg metric by $\mathcal{H}_{\mathrm{H}}^{s}$ and $\operatorname{dim}_{\mathrm{H}}$. The Hausdorff measure and dimension with respect to the Euclidean metric are denoted by $\mathcal{H}_{\mathrm{E}}^{s}$ and $\operatorname{dim}_{\mathrm{E}}$.

In this paper we consider projections onto homogeneous subgroups of $\mathbb{H}^{n}$. A homogeneous subgroup $\mathbb{G}$ of $\mathbb{H}^{n}$ is a subgroup which is closed under the intrinsic dilatations $\delta_{s}(z, t)=\left(s z, s^{2} t\right), s>0$. There are two kinds of homogeneous subgroups of $\mathbb{H}^{n}$. The horizontal subgroups are the ones which are contained in $\mathbb{R}^{2 n} \times\{0\}$ and the vertical subgroups are the ones which contain the $t$-axis $\{0\} \times \mathbb{R}$. Horizontal subgroups can be identified with linear subspaces of $\mathbb{R}^{2 n+1}$ which are contained 
in $\mathbb{R}^{2 n} \times\{0\}$. However, not every linear subspace of this form is a horizontal subgroup, only those corresponding to isotropic subspaces $V$ of $\mathbb{R}^{2 n}$ are. The restriction of the Heisenberg metric to a horizontal subgroup coincides with the Euclidean metric and therefore it is not necessary to specify the metric used in computing the Hausdorff measure or Hausdorff dimension of a subset of a horizontal subgroup. In this case we denote the Hausdorff measure and Hausdorff dimension simply by $\mathcal{H}^{s}$ and $\operatorname{dim}$.

Let $\mathbb{V}=V \times\{0\}$ be a horizontal subgroup. Consider $\mathbb{V}^{\perp}=V^{\perp} \times \mathbb{R}$, where $V^{\perp}$ is the orthogonal complement of $V$ in $\mathbb{R}^{2 n}$. Then $\mathbb{V}^{\perp}$ is a vertical subgroup of $\mathbb{H}^{n}$ and it will be called the vertical subgroup associated to $V$. Each point $p \in \mathbb{H}^{n}$ can be written uniquely as

$$
p=P_{\mathbb{V} \perp}(p) * P_{\mathbb{V}}(p),
$$

with $P_{\mathbb{V} \perp}(p) \in \mathbb{V}^{\perp}$ and $P_{\mathbb{V}}(p) \in \mathbb{V}$. This gives rise to a well-defined horizontal projection

$$
P_{\mathbb{V}}: \mathbb{H}^{n} \rightarrow \mathbb{V},(z, t) \mapsto P_{\mathbb{V}}(z, t)=\left(P_{V}(z), 0\right),
$$

and a vertical projection

$$
P_{\mathbb{V} \perp}: \mathbb{H}^{n} \rightarrow \mathbb{V}^{\perp},(z, t) \mapsto P_{\mathbb{V} \perp}(z, t)=\left(P_{V^{\perp}}(z), t-2 \omega\left(P_{V^{\perp}}(z), P_{V}(z)\right)\right) .
$$

Since there is a one-to-one correspondence between isotropic subspaces of $\mathbb{R}^{2 n}$ and horizontal subgroups of $\mathbb{H}^{n}$, these projections may be parametrized by the isotropic Grassmannian $G_{h}(n, m)$. For more information on the projections of the Heisenberg group, see [1] and [2].

We denote the family of all horizontal projections onto $m$-dimensional subgroups by $\mathcal{F}_{h}(n, m)$ and the corresponding projections in the Euclidean space $\mathbb{R}^{2 n}$ by $\mathcal{F}_{h}^{2 n}(n, m)$. That is,

$$
\mathcal{F}_{h}(n, m)=\left\{P_{\mathbb{V}}: \mathbb{H}^{n} \rightarrow \mathbb{V}: V \in G_{h}(n, m)\right\}
$$

and

$$
\mathcal{F}_{h}^{2 n}(n, m)=\left\{P_{V}: \mathbb{R}^{2 n} \rightarrow V: V \in G_{h}(n, m)\right\} .
$$

Note that when $m>1$, the family $\mathcal{F}_{h}^{2 n}(n, m)$ has dimension $2 n m-m(3 m-1) / 2$ $<m(2 n-m)$, and therefore one cannot apply standard projection theorems (e.g., Marstrand's projection theorem or the Besicovitch-Federer projection theorem) to obtain dimension results for these projections.

\section{Transversality}

In this section we show that the family $\mathcal{F}_{h}^{2 n}(n, m)$ of projections is transversal for every $0<m \leq n$. We begin with the definition of transversality.

Definition 3.1. Let $\Lambda \subset \mathbb{R}^{l}$ be open. A family of maps $\left\{\pi_{\lambda}: \mathbb{R}^{n} \rightarrow \mathbb{R}^{m}\right\}_{\lambda \in \Lambda}$ is transversal if it satisfies the following conditions for each compact set $K \subset \mathbb{R}^{n}$ :

(1) The mapping $\pi: \Lambda \times K \rightarrow \mathbb{R}^{m},(\lambda, x) \mapsto \pi_{\lambda}(x)$, is continuously differentiable and twice differentiable with respect to $\lambda$. 
(2) For $j=1,2$ there exist constants $C_{j}$ such that the derivatives with respect to $\lambda$ satisfy

$$
\left\|D_{\lambda}^{j} \pi(\lambda, x)\right\| \leq C_{j} \quad \text { for all }(\lambda, x) \in \Lambda \times K
$$

(3) For all $\lambda \in \Lambda$ and $x, y \in K$ with $x \neq y$, define

$$
\Phi_{x, y}(\lambda)=\frac{\pi_{\lambda}(x)-\pi_{\lambda}(y)}{\|x-y\|} .
$$

Then there exists a constant $C_{T}>0$ such that the property

$$
\left\|\Phi_{x, y}(\lambda)\right\| \leq C_{T}
$$

implies that

$$
\operatorname{det}\left(D_{\lambda} \Phi_{x, y}(\lambda)\left(D_{\lambda} \Phi_{x, y}(\lambda)\right)^{T}\right) \geq C_{T}^{2}
$$

(4) There exists a constant $C_{L}$ such that

$$
\left\|D_{\lambda}^{2} \Phi_{x, y}(\lambda)\right\| \leq C_{L}
$$

for all $\lambda \in \Lambda$ and $x, y \in K, x \neq y$.

Remark 3.2. Note that in the definition of transversality the target space of the mappings is fixed. In the case of the family $\mathcal{F}_{h}^{2 n}(n, m)$ this is not the case. However, identifying each $m$-plane $V$ with $\mathbb{R}^{m}$ we can think of the maps $P_{V}$ as mappings $\mathbb{R}^{n} \rightarrow \mathbb{R}^{m}$. Moreover, in the definition the maps are parametrized by $\Lambda \subset \mathbb{R}^{l}$, but the parameter space of $\mathcal{F}_{h}^{2 n}(n, m)$ is the manifold $G_{h}(n, m)$. Transversality is a local property and by saying that the family $\mathcal{F}_{h}^{2 n}(n, m)$ is transversal we mean that for each $V \in G_{h}(n, m)$ there exists a coordinate neighbourhood of $V$ such that Definition 3.1 holds in local coordinates.

Remark 3.3. If $m=1$, the isotropic Grassmannian $G_{h}(n, 1)$ and the Grassmann manifold $G(2 n, 1)$ are the same, $G_{h}(n, 1)=G(2 n, 1)$. In this case the family of isotropic projections is the same as the family of all orthogonal projections onto 1-dimensional subspaces, which is known to be transversal (see the remark after Theorem 7.3 in [9]). Thus for the rest of this section we assume that $m \geq 2$.

Transversal projection families have many useful properties. For instance, the Hausdorff dimensions of sets and measures are preserved under almost all projections. The theory of transversal mappings was extensively studied by Peres and Schlag in [9]. A recent result for transversal projection families that we will use in this paper is the Besicovitch-Federer projection theorem. See [5] for the proof.

Theorem 3.4. Let $E \subset \mathbb{R}^{n}$ be $\mathcal{H}^{m}$-measurable with $\mathcal{H}^{m}(E)<\infty$. Assume that $\Lambda \subset \mathbb{R}^{l}$ is open and $\left\{\pi_{\lambda}: \mathbb{R}^{n} \rightarrow \mathbb{R}^{m}\right\}_{\lambda \in \Lambda}$ is a transversal family of maps. Then $E$ is purely $m$-unrectifiable if and only if $\mathcal{H}^{m}\left(\pi_{\lambda}(E)\right)=0$ for $\mathcal{L}^{l}$-almost all $\lambda \in \Lambda$. 
Fix $0<m \leq n$. To show that the transversality condition holds locally, we fix an $m$-plane $V \in G_{h}(n, m)$, take a coordinate system around $V$, and show that the transversality conditions hold in this coordinate neighbourhood.

Define a family of projections $\pi: \mathcal{M}_{h}(n, m) \times \mathbb{R}^{2 n} \rightarrow \mathbb{R}^{m}$ by

$$
\begin{aligned}
\pi(A, x)=\pi_{A}(x) & =\left(\left(e_{1}^{A} \mid x\right), \ldots,\left(e_{m}^{A} \mid x\right)\right) \\
& =\left(x_{1}+\sum_{k=1}^{2 n-m} a_{k 1} x_{k+m}, \ldots, x_{m}+\sum_{k=1}^{2 n-m} a_{k m} x_{k+m}\right) .
\end{aligned}
$$

The projection $\pi_{A}$ is not quite the same as the orthogonal projection $P_{V_{A}}$ onto the $m$-plane $V_{A}$ corresponding to the matrix $A$, since the basis $\left\{e_{1}^{A}, \ldots, e_{m}^{A}\right\}$ of $V_{A}$ is not orthonormal, but we will see that for $A$ close to 0 the projections are sufficiently close.

Define the mapping $\Phi:\left\{(A, x, y) \in \mathcal{M}_{h}(n, m) \times \mathbb{R}^{2 n} \times \mathbb{R}^{2 n}: x \neq y\right\} \rightarrow \mathbb{R}^{m}$ by

$$
\Phi(A, x, y)=\Phi_{x, y}(A)=\frac{\pi_{A}(x)-\pi_{A}(y)}{\|x-y\|} .
$$

Writing $b=\|x-y\|^{-1}(x-y) \in S^{2 n-1}$ and using the linearity of the projection $\pi_{A}$ we see that

$$
\Phi_{x, y}(A)=\frac{\pi_{A}(x)-\pi_{A}(y)}{\|x-y\|}=\frac{\pi_{A}(x-y)}{\|x-y\|}=\pi_{A}\left(\frac{x-y}{\|x-y\|}\right)=\pi_{A}(b)=: \Phi_{b}(A) .
$$

We will show that, for the family defined above, there holds

$$
\operatorname{det}\left(D_{A} \Phi_{x, y}(0)\left(D_{A} \Phi_{x, y}(0)\right)^{T}\right) \geq \frac{1}{2}\left(1-\left\|\Phi_{x, y}(0)\right\|^{2}\right)^{m},
$$

for every $x, y \in \mathbb{R}^{2 n}$ with $x \neq y$.

Before we prove this inequality, we show that it implies that the family $\mathcal{F}_{h}^{2 n}(n, m)$ is locally transversal. The family clearly satisfies the conditions (1), (2), and (4) in Definition 3.1, so we need to show that the condition (3) is also satisfied. We examine the problem in the local coordinates $(U, \varphi)$ defined above and show that there exists a neighbourhood of 0 in which the transversality condition (3) holds. Let $\left\{v_{1}^{A}, \ldots, v_{m}^{A}\right\}$ be the orthonormal basis obtained by applying the GramSchmidt algorithm to the basis $\left\{e_{1}^{A}, \ldots, e_{m}^{A}\right\}$. Then, at $A=0$, we have, for every $b \in S^{2 n-1}$,

$$
\left(v_{i}^{0} \mid b\right)=\left(e_{i}^{0} \mid b\right)
$$

and

$$
\left.\partial_{\alpha \beta}\right|_{A=0}\left(v_{i}^{A} \mid b\right)=\left.\partial_{\alpha \beta}\right|_{A=0}\left(e_{i}^{A} \mid b\right),
$$

for all $i, \beta=1, \ldots, m$, and $\alpha \in\{1, \ldots, n-m+\beta, n+1, \ldots, 2 n-m\}$, where $\partial_{\alpha \beta}$ denotes the partial derivative with respect to the entry $a_{\alpha \beta}$ in the matrix $A=\left(a_{\alpha \beta}\right)$. Equation (3.2) is clear and the equation (3.3) follows from the fact that the GramSchmidt algorithm produces second order terms whose derivatives vanish at $A=0$. 
Defining $\Phi^{\mathcal{F}}:\left\{(A, x, y) \in \mathcal{M}_{h}(n, m) \times \mathbb{R}^{2 n} \times \mathbb{R}^{2 n}: x \neq y\right\} \rightarrow \mathbb{R}^{m}$ by

$$
\Phi^{\mathcal{F}}(A, x, y)=\Phi_{x, y}^{\mathcal{F}}\left(V_{A}\right)=\frac{P_{V_{A}}(x)-P_{V_{A}}(y)}{\|x-y\|}
$$

equations (3.2) and (3.3) imply that at $A=0$,

$$
\Phi_{x, y}^{\mathcal{F}}\left(V_{0}\right)=\Phi_{x, y}(0)
$$

and

$$
\operatorname{det}\left(D_{A} \Phi_{x, y}(0)\left(D_{A} \Phi_{x, y}(0)\right)^{T}\right)=\operatorname{det}\left(D_{A} \Phi_{x, y}^{\mathcal{F}}\left(V_{0}\right)\left(D_{A} \Phi_{x, y}^{\mathcal{F}}\left(V_{0}\right)\right)^{T}\right)
$$

for every $x, y \in \mathbb{R}^{2 n}$ such that $x \neq y$. We also use the notation $\widetilde{\Phi}^{\mathcal{F}}(A, b)=$ $\widetilde{\Phi}_{b}^{\mathcal{F}}\left(V_{A}\right)=P_{V_{A}}(b)$ for $b \in S^{2 n-1}$. Note that $\widetilde{\Phi}^{\mathcal{F}}(A,(x-y) /\|x-y\|)=\Phi^{\mathcal{F}}(A, x, y)$. The functions $\Phi^{\mathcal{F}}$ and $\widetilde{\Phi}^{\mathcal{F}}$ are smooth, so defining $\mathcal{M}_{h}^{1}(n, m)=\mathcal{M}_{h}(n, m) \cap B(0,1)$, we may choose a Lipschitz constant $L_{1} \geq 1$ for $\widetilde{\Phi}^{\mathcal{F}}$ and a Lipschitz constant $L_{2} \geq 1$ for

$$
(A, b) \mapsto \operatorname{det}\left(D_{A} \widetilde{\Phi}_{b}^{\mathcal{F}}\left(V_{A}\right)\left(D_{A} \widetilde{\Phi}_{b}^{\mathcal{F}}\left(V_{A}\right)\right)^{T}\right) \quad \text { on } \mathcal{M}_{h}^{1}(n, m) \times S^{2 n-1}
$$

Let

$$
0<C_{T} \leq 2^{-(m+2) / 2} \quad \text { and } \quad \epsilon=\min \left\{\frac{C_{T}}{L_{1}}, \frac{\left(1-4 C_{T}^{2}\right)^{m}}{4 L_{2}}\right\}
$$

If $(A, b) \in\left(\mathcal{M}_{h}(n, m) \cap B(0, \epsilon)\right) \times S^{2 n-1}$ is such that

$$
\left\|\widetilde{\Phi}_{b}^{\mathcal{F}}\left(V_{A}\right)\right\| \leq C_{T}
$$

we have, by (3.2),

$$
\left\|\Phi_{b}(0)\right\|=\left\|\widetilde{\Phi}_{b}^{\mathcal{F}}\left(V_{0}\right)\right\| \leq L_{1} \epsilon+C_{T} \leq 2 C_{T}
$$

and, by (3.1) and (3.3),

$$
\begin{aligned}
\operatorname{det}\left(D_{A} \widetilde{\Phi}_{b}^{\mathcal{F}}\left(V_{0}\right)\left(D_{A} \widetilde{\Phi}_{b}^{\mathcal{F}}\left(V_{0}\right)\right)^{T}\right) & =\operatorname{det}\left(D_{A} \Phi_{b}(0)\left(D_{A} \Phi_{b}(0)\right)^{T}\right) \\
& \geq \frac{1}{2}\left(1-\left\|\Phi_{b}(0)\right\|^{2}\right)^{m} \geq \frac{1}{2}\left(1-4 C_{T}^{2}\right)^{m}
\end{aligned}
$$

This implies that

$$
\operatorname{det}\left(D_{A} \widetilde{\Phi}_{b}^{\mathcal{F}}\left(V_{A}\right)\left(D_{A} \widetilde{\Phi}_{b}^{\mathcal{F}}\left(V_{A}\right)\right)^{T}\right) \geq \frac{1}{2}\left(1-4 C_{T}^{2}\right)^{m}-L_{2} \epsilon \geq \frac{1}{4}\left(1-4 C_{T}^{2}\right)^{m} \geq C_{T}^{2}
$$

by the choice of $C_{T}$ and $\epsilon$. We have shown that, assuming inequality (3.1), every plane $V \in G_{h}(n, m)$ has a coordinate neighbourhood on which the family $\mathcal{F}_{h}^{2 n}(n, m)$ satisfies the transversality condition. Next we prove the inequality (3.1). 
Recalling (2.2), we see that for $A \in \mathcal{M}_{h}(n, m)$ the $j$ th component function of the projection $\pi_{A}$ has the form

$$
\begin{aligned}
\pi_{A}^{j}(x)= & x_{j}+\sum_{k=1}^{2 n-m} a_{k j} x_{m+k} \\
= & x_{j}+\sum_{k=1}^{n-m+j} a_{k j} x_{m+k}+\sum_{k=n+1}^{2 n-m} a_{k j} x_{m+k} \\
& +\sum_{k=j+1}^{m}\left(a_{(n-m+j) k}+\sum_{l=1}^{n-m}\left(a_{l j} a_{(l+n) k}-a_{l k} a_{(l+n) j}\right)\right) x_{n+k} .
\end{aligned}
$$

The partial derivatives with respect to the entries in the matrix $A$ are

$$
\partial_{\alpha \beta} \pi_{A}^{j}(x)= \begin{cases}x_{m+\alpha}+\sum_{k=j+1}^{m} a_{(\alpha+n) k} x_{n+k}, & \text { for } \beta=j, 1 \leq \alpha \leq n-m, \\ & \text { for } \beta=j, \\ x_{m+\alpha}, & n-m+1 \leq \alpha \leq n-m+j, \\ x_{m+\alpha}-\sum_{k=j+1}^{m} a_{(\alpha-n) k} x_{n+k}, & \text { for } \beta=j, n+1 \leq \alpha \leq 2 n-m, \\ -a_{(\alpha+n) j} x_{n+\beta}, & \text { for } \beta>j, 1 \leq \alpha \leq n-m, \\ x_{n+\beta}, & \text { for } \beta>j, \alpha=n-m+j, \\ a_{(\alpha-n) j} x_{n+\beta}, & \text { for } \beta>j, n+1 \leq \alpha \leq 2 n-m, \\ 0, & \text { elsewhere. }\end{cases}
$$

From this we may compute the matrix $B_{A, x}=D_{A} \pi_{A}(x)\left(D_{A} \pi_{A}(x)\right)^{T}$ at $A=0$. The matrix $D_{A} \pi_{A}(x)$ is an $m \times(2 n m-m(3 m-1) / 2)$-matrix. The rows correspond to the component functions of the mapping $\pi_{A}$ and the columns correspond to all possible pairs $(\alpha, \beta)$. When $A=0$, the entries of the $j$ th row of the matrix $D_{A} \pi_{A}(x)$ are

$$
\begin{array}{ll}
x_{m+\alpha}, & \text { for } \beta=j, \alpha \in\{1, \ldots, n-m+j, n+1, \ldots, 2 n-m\}, \\
x_{n+\beta}, & \text { for } \alpha=n-m+j, \beta \in\{j+1, \ldots, m\}, \\
0, & \text { elsewhere. }
\end{array}
$$

From this we see that the coordinates $x_{m+1}, \ldots, x_{2 n}$ appear on each row exactly once and the other entries are zero. Thus all the diagonal entries of the matrix $B_{0, x}$ are $\left[B_{0, x}\right]_{i i}=\Sigma_{k=1}^{2 n-m} x_{m+k}^{2}$ for every $i=1, \ldots, m$.

The entries $x_{m+1}, \ldots, x_{2 n}$ appear on different positions on different rows. If $i<j$, there is a nonzero entry in both rows $i$ and $j$ of the same column if and only if $\alpha=n-m+i$ and $\beta=j$. The entry of the $i$ th row is $x_{n+\beta}=x_{n+j}$ and the entry of the $j$ th row is $x_{m+\alpha}=x_{n+i}$. This implies that the off-diagonal entries of 
the matrix $B_{0, x}$ are $\left[B_{0, x}\right]_{i j}=x_{n+i} x_{n+j}$ for every $i, j=1, \ldots, m, i \neq j$.

$$
\left[B_{0, x}\right]_{i j}= \begin{cases}\sum_{k=1}^{2 n-m} x_{m+k}^{2}=: \Delta_{x}, & \text { for } i=j \\ x_{n+i} x_{n+j} & \text { for } i \neq j .\end{cases}
$$

Using an inductive argument one can see that the determinant of this matrix is

$$
\operatorname{det} B_{0, x}=\Delta_{x}^{m}+\sum_{i=2}^{m}(-1)^{i-1}(i-1) \Delta_{x}^{m-i} \sum_{\alpha \in \Lambda(m, i)} x_{\alpha(1)}^{2} \cdots x_{\alpha(i)}^{2},
$$

where

$$
\Lambda(m, i)=\{\alpha=(\alpha(1), \ldots, \alpha(i)): \alpha(k) \in\{n+1, \ldots, n+m\} \forall k, \alpha(1)<\ldots<\alpha(i)\}
$$

is the set of all strictly increasing sequences of length $i$ consisting of integers from the interval $[n+1, n+m]$.

Let $b \in S^{2 n-1}$. Note that the entry $\Delta_{b}$ on the diagonal of the matrix $B_{0, b}$ is precisely $\left\|P_{V_{0}^{\perp}}(b)\right\|^{2}=1-\left\|\Phi_{b}(0)\right\|^{2}$. We will show that

$$
\operatorname{det} B_{0, b} \geq \Delta_{b}^{m}-\Delta_{b}^{m-2} \sum_{\alpha \in \Lambda(m, 2)} b_{\alpha(1)}^{2} b_{\alpha(2)}^{2} \geq \frac{1}{2} \Delta_{b}^{m} .
$$

The second inequality is easy:

$$
\begin{aligned}
\frac{1}{2} \Delta_{b}^{m}-\Delta_{b}^{m-2} \sum_{\alpha \in \Lambda(m, 2)} b_{\alpha(1)}^{2} b_{\alpha(2)}^{2} & \geq \frac{1}{2} \Delta_{b}^{m-2}\left(\left(\sum_{i=1}^{m} b_{n+i}^{2}\right)^{2}-\sum_{\alpha \in \Lambda(m, 2)} 2 b_{\alpha(1)}^{2} b_{\alpha(2)}^{2}\right) \\
& =\frac{1}{2} \Delta_{b}^{m-2} \sum_{i=1}^{m} b_{n+i}^{4} \geq 0 .
\end{aligned}
$$

If $m=2$, the first inequality holds clearly as an equality. If $m=3$, the first inequality is also clear since one only removes one positive term from the determinant. Thus we may assume that $m \geq 4$. It is enough to show that for any $i \in\{3, \ldots, m-1\}$ there holds

$$
(i-1) \Delta_{b}^{m-i} \sum_{\alpha \in \Lambda(m, i)} b_{\alpha(1)}^{2} \cdots b_{\alpha(i)}^{2} \geq i \Delta_{b}^{m-i-1} \sum_{\alpha \in \Lambda(m, i+1)} b_{\alpha(1)}^{2} \cdots b_{\alpha(i+1)}^{2} .
$$

Using the fact that $\Delta_{b} \geq \sum_{j=n+1}^{n+m} b_{j}^{2}$, we see that the above inequality holds if

$$
(i-1) \sum_{\alpha \in \Lambda(m, i)} b_{\alpha(1)}^{2} \cdots b_{\alpha(i)}^{2}\left(\sum_{j=n+1}^{n+m} b_{j}^{2}\right) \geq i \sum_{\alpha \in \Lambda(m, i+1)} b_{\alpha(1)}^{2} \cdots b_{\alpha(i+1)}^{2} .
$$


Now

$$
\begin{aligned}
& (i-1) \sum_{\alpha \in \Lambda(m, i)} b_{\alpha(1)}^{2} \cdots b_{\alpha(i)}^{2}\left(\sum_{j=n+1}^{n+m} b_{j}^{2}\right)-i \sum_{\alpha \in \Lambda(m, i+1)} b_{\alpha(1)}^{2} \cdots b_{\alpha(i+1)}^{2} \\
& =(i-1) \sum_{\alpha \in \Lambda(m, i)} b_{\alpha(1)}^{2} \cdots b_{\alpha(i)}^{2}\left(\sum_{j=n+1}^{\alpha(i)} b_{j}^{2}\right) \\
& -\sum_{\alpha \in \Lambda(m-1, i)} b_{\alpha(1)}^{2} \cdots b_{\alpha(i)}^{2}\left(\sum_{k=\alpha(i)+1}^{n+m} b_{k}^{2}\right) \\
& =(i-1) \sum_{k=n+i}^{n+m} \sum_{j=n+1}^{k} \sum_{\alpha \in \Lambda(k-1, i-1)} b_{\alpha(1)}^{2} \cdots b_{\alpha(i-1)}^{2} b_{j}^{2} b_{k}^{2} \\
& -\sum_{j=n+i}^{n+m-1} \sum_{k=j+1}^{n+m} \sum_{\alpha \in \Lambda(j-1, i-1)} b_{\alpha(1)}^{2} \cdots b_{\alpha(i-1)}^{2} b_{k}^{2} b_{j}^{2} \\
& \geq \sum_{k=n+i+1}^{n+m} b_{k}^{2}\left(\sum_{j=n+1}^{k} \sum_{\alpha \in \Lambda(k-1, i-1)} b_{\alpha(1)}^{2} \cdots b_{\alpha(i-1)}^{2} b_{j}^{2}\right. \\
& \left.-\sum_{j=n+i}^{k-1} \sum_{\alpha \in \Lambda(j-1, i-1)} b_{\alpha(1)}^{2} \cdots b_{\alpha(i-1)}^{2} b_{j}^{2}\right) \\
& \geq 0 \text {, }
\end{aligned}
$$

which proves the first inequality in (3.4). This shows that the inequality (3.1) is valid and finishes the proof that the family $\mathcal{F}_{h}^{2 n}(n, m)$ is transversal.

\section{Applications}

Transversality together with Theorem 3.4 implies that the Besicovitch-Federer projection theorem holds for isotropic projections.

Theorem 4.1. Let $E \subset \mathbb{R}^{2 n}$ be $\mathcal{H}^{m}$-measurable with $\mathcal{H}^{m}(E)<\infty$. Then $E$ is purely m-unrectifiable if and only if $\mathcal{H}^{m}\left(P_{V}(E)\right)=0$ for $\mu_{n, m}$-almost all $V \in$ $G_{h}(n, m)$.

The $\alpha$-energy $I_{\alpha}(\mu)$ of a measure $\mu$ on $\mathbb{R}^{n}$ is defined by

$$
I_{\alpha}(\mu)=\iint|x-y|^{-\alpha} d \mu y d \mu x .
$$

and the Sobolev dimension of a finite measure $\mu$ on $\mathbb{R}^{n}$ is defined by

$$
\operatorname{dim}_{\mathrm{S}} \mu=\sup \left\{\alpha \in \mathbb{R}: \int(1+|x|)^{\alpha-n}|\hat{\mu}(x)|^{2} d x<\infty\right\},
$$

where $\hat{\mu}$ is the Fourier transform of the measure $\mu$. 
Transversality immediately yields also the following result concerning the dimension of projected measures. See Theorem 7.3 in [9] for more information.

Theorem 4.2. Let $0<m \leq n$ and suppose that $\mu$ is a finite positive measure on $\mathbb{R}^{2 n}$ with finite $\alpha$-energy for some $\alpha>0$. If $\sigma \in(0, \alpha]$, then

$$
\operatorname{dim}\left\{V \in G_{h}(n, m): \operatorname{dim}_{S} \mu_{V}<\sigma\right\} \leq 2 n m-\frac{m(3 m-1)}{2}+\sigma-\max \{\alpha, m\},
$$

where $\operatorname{dim}_{\mathrm{S}}$ is the Sobolev dimension of a measure and $\mu_{V}$ is the projection of the measure $\mu$ onto the m-plane $V$, that is, $\mu_{V}(A)=\mu\left(P_{V}^{-1}(A)\right)$ for all $A \subset V$.

It follows from the definition of Sobolev dimension that if $0<\operatorname{dim}_{\mathrm{S}} \mu \leq n$, then $\operatorname{dim}_{\mathrm{S}} \mu=\sup \left\{\alpha: I_{\alpha}(\mu)<\infty\right\}$. In particular, if a Borel set $E \subset \mathbb{R}^{n}$ supports a probability measure $\mu$ with $\operatorname{dim}_{\mathrm{S}}(\mu) \leq n$, then $\operatorname{dim} E \geq \operatorname{dim}_{\mathrm{S}} \mu$. If $\operatorname{dim}_{\mathrm{S}} \mu>n$, then $\mu$ is absolutely continuous. These facts together with Theorem 4.2 imply the following result on the dimension of exceptional sets.

Theorem 4.3. Let $n$ and $m$ be integers such that $0<m \leq n$ and let $E \subset \mathbb{R}^{2 n}$ be a Borel set with $\operatorname{dim} E=s$.

(1) If $s \leq m$, then

$$
\begin{aligned}
\operatorname{dim}\left\{V \in G_{h}(n, m): \operatorname{dim} P_{V}(E)<s\right\} & \leq 2 n m-\frac{m(3 m+1)}{2}+s \\
& =\operatorname{dim} G_{h}(n, m)-(m-s)
\end{aligned}
$$

(2) If $s>m$, then

$$
\begin{aligned}
\operatorname{dim}\left\{V \in G_{h}(n, m): \mathcal{H}^{m}\left(P_{V}(E)\right)=0\right\} & \leq 2 n m-\frac{3 m(m-1)}{2}-s \\
& =\operatorname{dim} G_{h}(n, m)-(s-m)
\end{aligned}
$$

Proof. Assume first that $s>m$. Then by Frostman's lemma we can take $\alpha>m$ and a probability measure $\mu$ supported on $E$ such that $I_{\alpha}(\mu)<\infty$. Now Theorem 4.2 implies that

$$
\begin{aligned}
\operatorname{dim}\left\{V \in G_{h}(n, m): \mu_{V} \text { is not absolutely continuous }\right\} \\
\quad \leq \operatorname{dim}\left\{V \in G_{h}(n, m): \operatorname{dim}_{\mathrm{S}} \mu_{V} \leq m\right\} \leq 2 n m-\frac{3 m(m-1)}{2}-\alpha .
\end{aligned}
$$

It follows that

$$
\operatorname{dim}\left\{V \in G_{h}(n, m): \mathcal{H}^{m}\left(P_{V}(E)\right)=0\right\} \leq 2 n m-\frac{3 m(m-1)}{2}-\alpha .
$$

Letting $\alpha \nearrow s$ implies the claim. The first claim is proven similarly. 
In Theorem 1.2 of [2] it is shown that almost all isotropic projections onto $m$-planes preserve the Hausdorff dimension for sets whose dimension is at most $m$. For sets with dimension greater than $m$ almost all projections have positive $\mathcal{H}^{m}$ measure. The proof in [2] uses energy estimates and Frostman's lemma. Theorem 4.3 strengthens this theorem by providing dimension estimates for the sets of exceptional parameters.

The behaviour of dimensions of sets and measures under subfamilies of orthogonal projections has been studied recently by E. Järvenpää, M. Järvenpää, and T. Keleti in [6] and D. Oberlin in [8]. Their results, however, do not give anything new in our setting because the family we are studying is transversal.

Theorems 4.1 and 4.3 yield corresponding results for the horizontal projections in the Heisenberg group. We denote by $\pi: \mathbb{H}^{n} \rightarrow \mathbb{R}^{2 n}, \pi(z, t)=z$, the projection onto the first $2 n$ coordinates.

Corollary 4.4. Let $E \subset \mathbb{H}^{n}$ be a Borel set with $\mathcal{H}_{\mathrm{E}}^{m}(\pi(E))<\infty$. Then $\mathcal{H}^{m}\left(P_{\mathbb{V}}(E)\right)=0$ for $\mu_{n, m}$-almost all $V \in G_{h}(n, m)$ if and only if $E \subset A \times \mathbb{R}$, where $A \subset \mathbb{R}^{2 n}$ is purely $m$-unrectifiable in the Euclidean sense.

Corollary 4.5. Let $n$ and $m$ be integers such that $0<m \leq n$ and let $E \subset \mathbb{H}^{n}$ be a Borel set with $\operatorname{dim}_{\mathrm{H}} E=s$.

(1) If $s \leq m+2$, then

$$
\begin{aligned}
\operatorname{dim}\left\{V \in G_{h}(n, m): \operatorname{dim} P_{\mathbb{V}}(E)<s-2\right\} & \leq 2 n m-\frac{m(3 m+1)}{2}+s-2 \\
& =\operatorname{dim} G_{h}(n, m)-(m-s+2) .
\end{aligned}
$$

(2) If $s>m+2$, then

$$
\begin{aligned}
\operatorname{dim}\left\{V \in G_{h}(n, m): \mathcal{H}^{m}\left(P_{\mathbb{V}}(E)\right)=0\right\} & \leq 2 n m-\frac{3 m(m-1)}{2}-s+2 \\
& =\operatorname{dim} G_{h}(n, m)-(s-m-2) .
\end{aligned}
$$

Proof. Since $P_{\mathbb{V}}=P_{V} \circ \pi$, we have, by [2] (see the proof of Theorem 1.1 therein), that $\operatorname{dim}_{\mathrm{E}} \pi(E) \geq \operatorname{dim}_{\mathrm{H}} E-2=s-2$. The rest of the proof is the same as for Theorem 4.3.

\section{References}

[1] Balogh, Z. M., Cartagena, E. D., Fässler, K., Mattila, P. and Tyson, J. T.: The effect of projections on dimension in the Heisenberg group. Rev. Mat. Iberoam. 29 (2013), no. 2, 381-432.

[2] Balogh, Z. M., Fässler, K., Mattila, P. And Tyson, J. T.: Projection and slicing theorems in Heisenberg groups. Adv. Math. 231 (2012), no. 2, 569-604.

[3] Capogna, L., Danielli, D., Pauls, S. D. and Tyson, J. T.: An introduction to the Heisenberg group and the sub-Riemannian isoperimetric problem. Progress in Mathematics 259, Birkhäuser Verlag, Basel, 2007. 
[4] Eliashberg, Y. and Traynor, L.: Symplectic geometry and topology. IAS/Park City Mathematics Series, Amer. Math. Soc., 2006.

[5] Hovila, R., Järvenpä̈̈, E., Järvenpä̈̈, M. And Ledrappier F.: BesicovitchFederer projection theorem and geodesic flows on Riemann surfaces. Geom. Dedicata 161 (2012), 51-61.

[6] JÄrvenpä̈̈, E., JärvenpäÄ, M. And Keleti, T.: Hausdorff dimension and nondegenerate families of projections. To appear in J. Geom. Anal.

[7] Mattila, P.: Hausdorff dimension, projections, and the Fourier transform. Publ. Mat. 48 (2004), no. 1, 3-48.

[8] Oberlin, D. M.: Exceptional sets of projections, unions of $k$-planes, and associated transforms. To appear in Israel J. Math.

[9] Peres, Y. And Schlag, W.: Smoothness of projections, Bernoulli convolutions, and the dimensions of exceptions. Duke Math. J. 102 (2000), no. 2, 193-251.

Received June 13, 2012.

Risto Hovila: Department of Mathematics and Statistics, P.O. Box 68, 00014 University of Helsinki, Finland.

E-mail: risto.hovila@helsinki.fi

The research was supported by the Finnish Centre of Excellence in Analysis and Dynamics Research and by the Jenny and Antti Wihuri Foundation. 